\title{
Maritime Detection of Radiological/Nuclear Threats with Hybrid Imaging System
}

\author{
Anthony L. Hutcheson, Bernard F. Phlips, Eric A. \\ Wulf, Lee J. Mitchell, W. Neil Johnson \\ High Energy Space Environment Branch \\ U.S. Naval Research Laboratory \\ Washington, DC, USA
}

\author{
Byron E. Leas \\ SRA International, Inc. \\ Fairfax, VA, US
}

\begin{abstract}
Improved detection of weapons of mass destruction is one of the Science and Technology priorities of the Secretary of Defense for Fiscal Years 2013-2017. Unfortunately, the remote detection of special nuclear materials is difficult because the materials are not very radioactive, the radiation signatures decrease rapidly with distance, and faint sources of radiation can be obscured by naturally occurring and man-made radioactive sources. The Radiation Detection Section of the High Energy Space Environment Branch of the U.S. Naval Research Laboratory has developed the SuperMISTI stand-off detection system for maritime environments. The instrument was deployed at Norfolk Naval Station in July 2012 as part of the Manta technology demonstration to determine the on-water performance of the system. Detailed descriptions of the SuperMISTI system and its operation are given.
\end{abstract}

Keywords-special nuclear materials; stand-off detection; coded aperture; gamma detection; neutron detection; maritime detection

\section{INTRODUCTION}

The SuperMISTI detection system [1] is a hybrid detection, identification, and imaging system for sources of gamma-ray radiation at stand-off distances. The system was designed and built at the U.S. Naval Research Laboratory (NRL) in Washington, DC, and is based on the Mobile Imaging and Spectroscopic Threat Identification (MISTI) system [2] that was designed at NRL for the Department of Homeland Security (DHS) Domestic Nuclear Detection Office (DNDO) Stand-Off Radiation Detection System (SORDS) program [3]. The SuperMISTI system uses the high-resolution spectra of highpurity germanium (HPGe) detectors to detect and identify gamma-ray sources as well as coded aperture technology and lower-cost $\mathrm{NaI}$ detectors to image and localize the detected sources. The system utilizes a modular design to allow the detection/identification and the imaging/localization portions to be used separately or together depending on the situation. The SuperMISTI system has demonstrated detection, identification, and localization of gamma and neutron sources in a maritime environment at operationally relevant stand-off distances.

\section{SYSTEM DESCRIPTION}

The complete SuperMISTI system comprises two separate detection subsystems: a high-purity germanium (HPGe) detection/identification system and a NaI imaging/localization system. Each subsystem is housed in a separate $20-\mathrm{ft}$

Work conducted under the auspices of the Maritime Weapons of Mass Destruction Detection Program of the Office of Naval Research. refrigerated ISO container that provides humidity and temperature control, and each subsystem can run independently as well as together. Power for each subsystem is provided by an individual clip-on diesel generator capable of providing approximately 5 days of continuous operation. Each subsystem is equipped with a pair of global positioning system (GPS) receivers to determine location, speed, and orientation. External optical cameras provide digital photos of the area to be investigated/imaged. Electronics including iseg high voltage supplies [4], mesytec shaping amplifiers and peak-sensing VME digitizers [5], and on-board servers are mounted in standard electronics racks that are shock-mounted inside each ISO container. External Ethernet ports installed on the containers allow the system to be controlled externally via laptop or remotely via wireless communication. A detailed description of each individual subsystem follows.

\section{A. Detection/Identification}

The SuperMISTI detection/identification subsystem utilizes a pallet-mounted array of 24 HPGe detectors (average relative efficiency $=110 \%$ ) that are individually shielded from above, below, and the sides by 0.5 " lead plates. The palletized array is shown in Fig. 1. Foam bedding is used to protect each detector from vibrational and shock damage. Each HPGe detector is attached to a 7-L ORTEC liquid nitrogen (LN2) dewar [6] that is filled once daily via an automated fill system. This fill system eliminates the need for any "open" LN2 in the container and removes personnel from the dangers associated with using

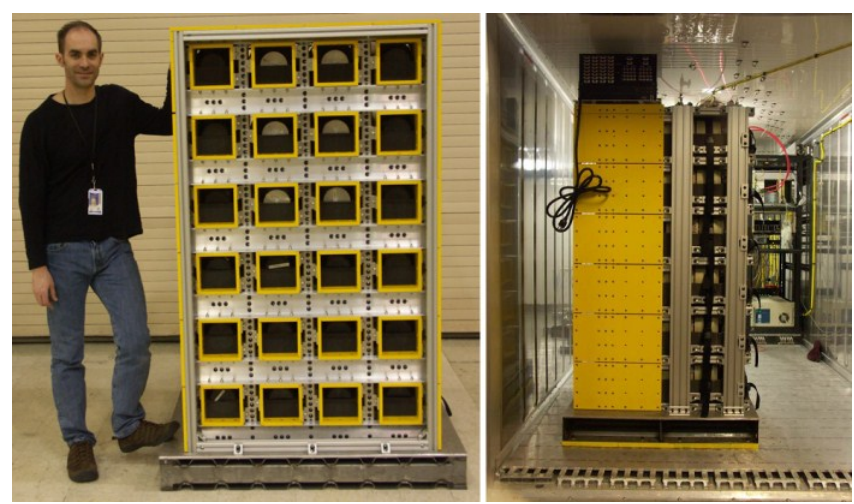

Figure 1. Pallet-mounted HPGe array. (left) Front view of array. (right) Side view of array installed in ISO container. 


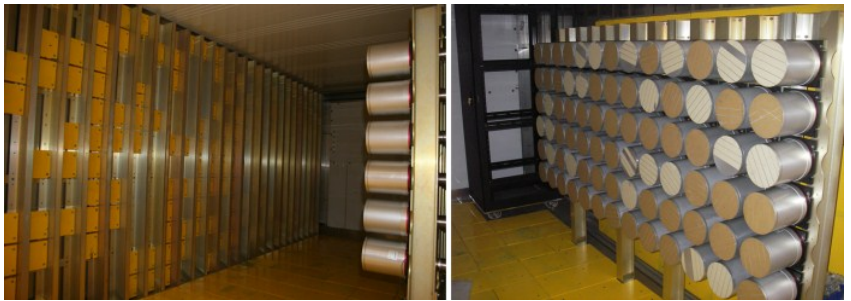

Figure 2. (Left) $12 \times 27$ lead coded aperture mounted to ISO container wall. (Right) $6 \times 13$ array of $\mathrm{NaI}$ detectors on opposite side of ISO container.

LN2. Each dewar has a cryogenic solenoid valve and a temperature sensor on the exhaust to monitor when the dewar is full. The valves and sensors are plate-mounted atop the palletized array. Separate sensors monitor the pressure on the input lines.

The combined weight of the detection/identification subsystem is approximately $17,000 \mathrm{lb}$.

\section{B. Imaging/Localization}

The SuperMISTI imaging/localization subsystem consists of $78 \mathrm{NaI}$ detectors (Ø6” $\times 6 ")$ mounted into a $6 \times 13$ array on one side of the ISO container. The entire array is shielded from above, below, and the sides by 1 " of lead; in addition, a 1" lead flooring provides further shielding from background radiation originating from below, and an additional layer of 1" lead on the back wall provides shielding from the rear. Mounted on the opposite side of the container is a $12 \times 27$ coded aperture that comprises 162 lead blocks $(6 " \times 6 " \times 2 ")$. The coded aperture and the detector array are shown in Fig. 2.

In addition to the gamma imaging detectors, a complement of six ${ }^{3} \mathrm{He}$ detectors (Ø5.8” $\left.\times 25.2 ” ; 2.66 \mathrm{~atm}\right)$ are integrated into the ISO container for neutron detection. These detectors are mounted near the end of the container and moderated by 1 " of high-density polyethylene on all sides.

The combined weight of the imaging/localization subsystem is approximately $32,000 \mathrm{lb}$.

\section{Operation}

When operated as a single detection system, the two subsystems are deployed together and connected via Ethernet. The system is started via a simple run GUI (graphical user interface) that records a unique run number, start and stop times, and a user-input run description. Real-time system monitoring is accomplished via a web GUI. The web GUI displays an overhead map/satellite image of the area with the truck location and, if applicable, detected source location overlaid. A rate window displays the count rates for any detected sources as well as for the standard ${ }^{40} \mathrm{~K}$ and ${ }^{226} \mathrm{Th}$ background lines. A spectrum window displays the current summed HPGe spectrum, and a status window alerts the user to any isotopes detected as well as the detection level and the integration time used. Finally, an optical camera image is displayed with, if applicable, an overlaid coded image of the detected source location. Currently, up to two source images can be displayed simultaneously on the optical camera image.

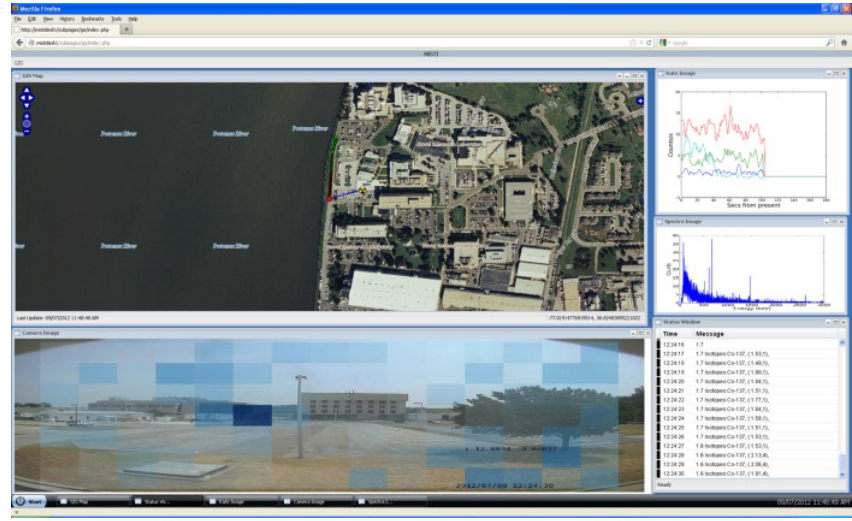

Figure 3. Web GUI displaying (clockwise from the top left) an overhead map/satellite imagery, the count rate window, the HPGe spectrum, the alarm/message window, and the overlaid camera image.

Source detection and identification are performed with the summed spectra from the HPGe detectors. Summed spectra are produced for integration times ranging from 1 to $120 \mathrm{~s}$. The summed spectra are analyzed using a region-of-interest (ROI) method. A local database of isotope photopeaks is used to define ROI windows as well as left and right background windows in each spectrum. The net counts in each ROI are calculate and used to determine a Poisson survival function that is compared with a threshold alarm level to determine isotope detection. Typically, the threshold alarm level is set based on the desired maximum allowable rate of "false positive" detections in a given time period. If an alarm is triggered, the identity of the isotope, the detection level, and the integration time are logged in the database, and the user is notified via the status window in the web GUI.

Once a source has been detected and identified, this information is used to determine a ROI in the NaI spectra; the width of this ROI is based on an assumed $8 \%$ resolution at 662 $\mathrm{keV}$. The counts in this ROI are used to produce a $7 \times 15$ coded image of the source. This coded image is then overlaid on an optical image from the external camera to produce a combined image that can show the source direction to the user; an example combined image is shown in Fig. 4.

A single coded image merely indicates a source direction; however, multiple such images taken while in motion allows the system to determine the three-dimensional (3D) location of the source. In practice, this is accomplished by dividing the $3 \mathrm{D}$

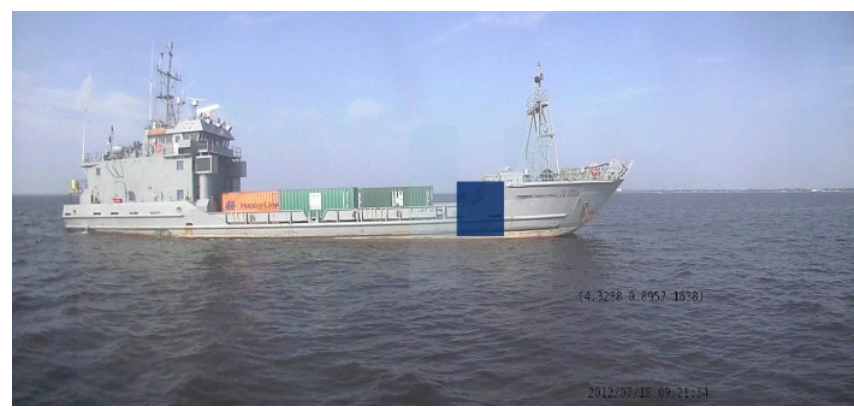

Figure 4. Reconstructed radiation source position image for a gamma-ray source located on a ship. The dark blue square indicates the source location. 

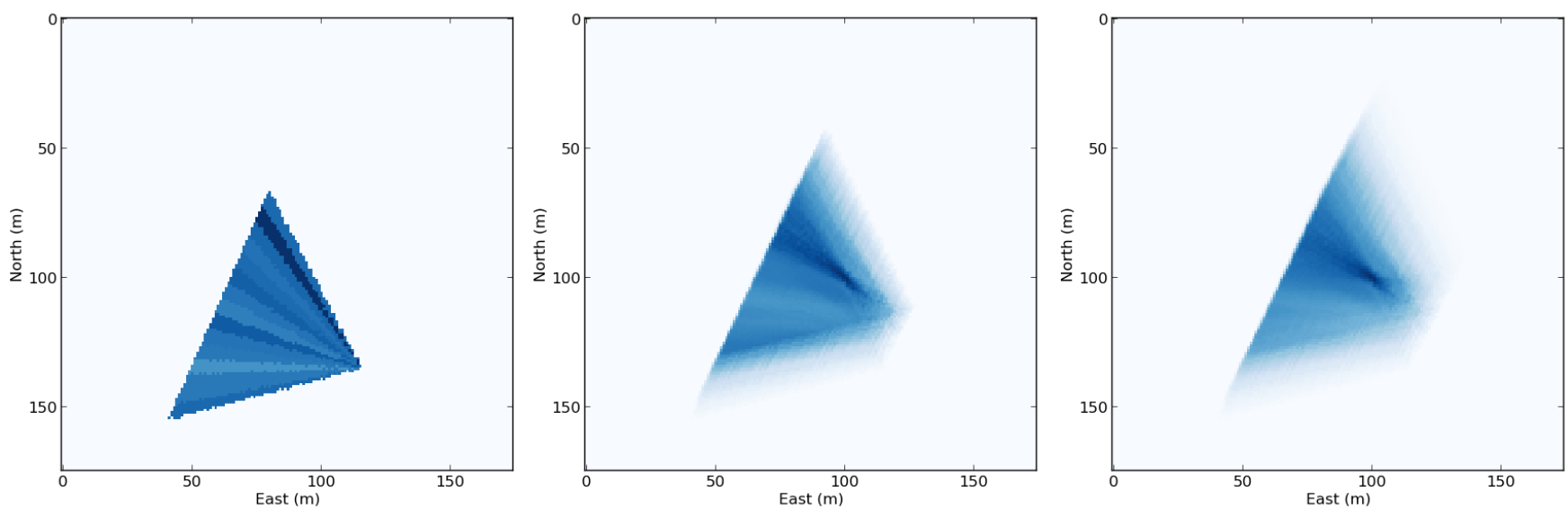

Figure 5. A 2D cross section of the 3D space populated by the projected coded image. The source location is approximately located at coordinates (100, 100). (Left) One coded image. (Center) 30 coded images. (Right) 60 coded images.

space into voxels and populating those voxels with counts based on the coded image for each time step. After sufficient time steps, the voxel with the greatest number of counts (the "hot spot") will indicate the source location. Using the location and orientation information of the system provided by the GPS receivers allows the GPS coordinates of the "hot spot" to be determined. This procedure is demonstrated in Fig. 5; in this figure, a horizontal cross section of the $3 \mathrm{D}$ space is plotted for one coded image, for 30 combined coded images, and for 60 combined coded images. In these images, the counts from the coded images are projected out only approximately $50 \mathrm{~m}$; in practice, these counts can be projected out hundreds of meters. Note that, as the number of coded images increase, the location of the source becomes more evident.

Source location for a neutron source is roughly determined by the perpendicular vector from the container's heading when the neutron counts are at their maximum value.

\section{MARITIME PERFORMANCE}

On 16-19 July 2012, the SuperMISTI detection system participated in the Manta technology demonstration organized by the Maritime Weapons of Mass Destruction Detection Program of the Office of Naval Research [7] and hosted at Naval Station Norfolk in Norfolk, VA. The aim of this demonstration was to determine the on-water performance of different detection systems. Each system was tasked with detecting

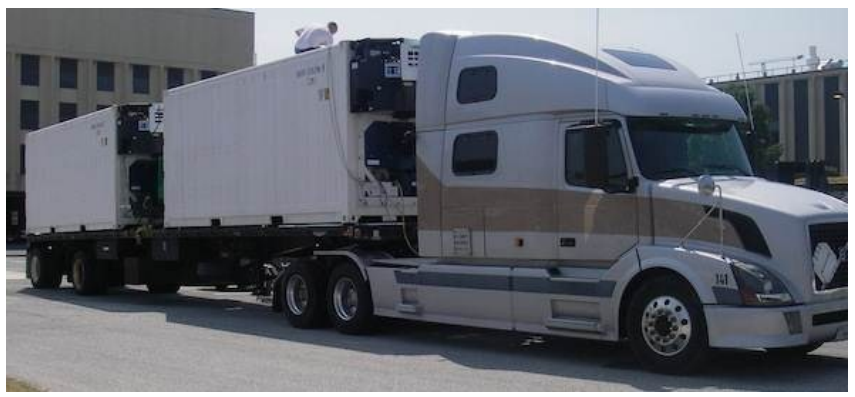

Figure 6 . The SuperMISTI system mounted on a 48 -ft trailer for testing prior to deployment to the Manta technology demonstration. various gamma and neutron sources that were hidden in different types of seagoing vessels that were either docked on a pier or moored in open water. The operational parameters for each system (speed, distance, etc.) were based on the expected performance of the system, which was determined prior to deployment.

\section{A. Predeployment Testing}

Prior to deployment to the Manta technology demonstration, the two SuperMISTI subsystems were mounted on a 48-ft trailer to simulate testing the combined system on a barge (see Fig. 6). The combined system was tested onsite at NRL with outdoor sources at speeds of up to $20 \mathrm{mph}$ and distances of up to $125 \mathrm{~m}$. The results of this testing were used with Monte Carlo modeling to estimate the system's performance in a maritime environment.

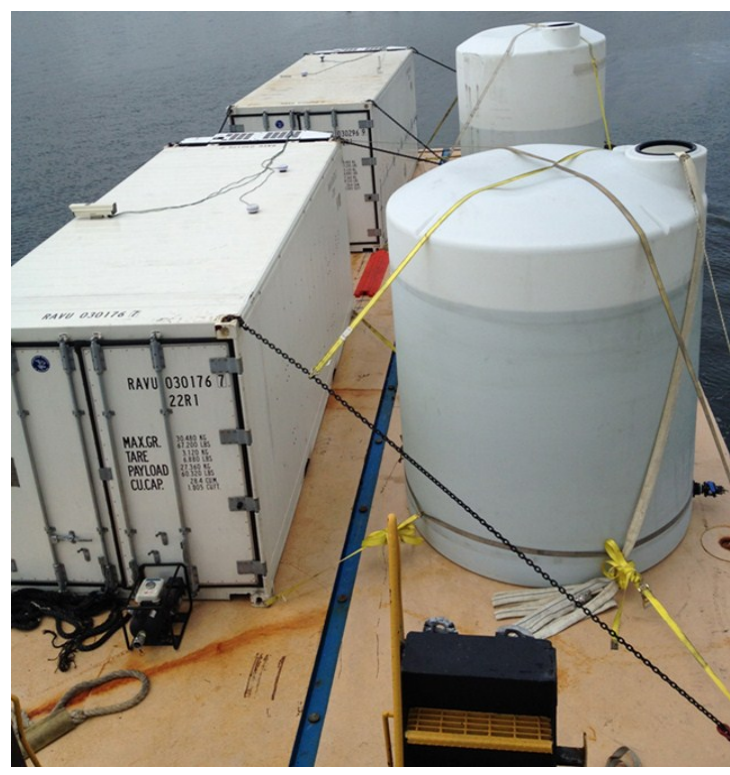

Figure 7. The complete SuperMISTI system on a $60-\mathrm{ft}$ barge. The water ballast tanks used to maintain a level barge are shown on the right. 


\section{B. Manta Technology Demonstration}

Both SuperMISTI subsystems were deployed on a $60-\mathrm{ft}$ barge that was pushed by a tug at speeds of up to $6 \mathrm{kn}$ and at stand-off distances of up to $300 \mathrm{~m}$. Water ballast tanks were utilized to maintain a level barge. The complete SuperMISTI system as deployed on the barge is shown in Fig. 7.

The results of this exercise were very close to the expected performance and clearly demonstrate the utility of the SuperMISTI system. All sources that were deployed in the demonstration were successfully detected, identified, and localized at operationally relevant distances ranging up to hundreds of meters.

\section{CONCLUSION}

The SuperMISTI hybrid system developed at NRL has demonstrated the successful detection, identification, and localization of gamma and neutron sources in a maritime environment at operationally relevant distances. Since the completion of the Manta demonstration, the number of HPGe detectors in the detection/identification subsystem has been increased from 24 to 48 , a modification that significantly enhances the gamma detection/identification capabilities of the system. Further performance enhancements planned for the future include the use of a large-area $\mathrm{BF}_{3}$ detector array to increase neutron detection capabilities and the implementation of better localization algorithms for neutron sources.

\section{ACKNOWLEDGMENT}

The authors of this work gratefully acknowledge the funding from the Maritime Weapons of Mass Destruction Detection Program of the Office of Naval Research.

\section{REFERENCES}

[1] E. A. Wulf, A. L. Hutcheson, B. F. Phlips, L. J. Mitchell and B. E. Leas, "Stand-off detection with an active interrogation photon beam," Nuclear Science Symposium and Medical Imaging Conference (NSS/MIC), 2011 IEEE, pp. 315-318, October 2011

[2] L. J. Mitchell et al., "Mobile imaging and spectroscopic threat identification (MISTI): system overview," Nuclear Science Symposium Conference Record (NSS/MIC), 2009 IEEE, pp. 110-118, October 2009.

[3] Domestic Nuclear Detection Office, Advanced Technology Demonstration of Stand-Off Radiation Detection Systems, BAA07-01, December 18, 2008.

[4] mesytec GmbH \& Co. KG, Wernher-von-Braun-Str. 1, 85640 Putzbrunn, Germany.

[5] iseg Spezialelektronik $\mathrm{GmbH}$, Bautzner Landstrasse 23, 01454 Radeberg, Germany.

[6] ORTEC, Advanced Measurement Technology, Inc., 801 South Illinois Avenue, Oak Ridge, TN.

[7] P. Morrison, "Maritime Weapons of Mass Destruction Detection," 2012 IEEE International Conference on Technologies for Homeland Security, November 2012, unpublished. 